\title{
SEARCH FOR SIGNS OF A NEW OUTBURST IN THE QUIESCENT STATE OF CH CYG
}

\author{
D. KOTNIK-KARUZA AND R. JURDANA ŠEPIĆ \\ Department of Physics - Faculty of Education,Rijeka, Croatia \\ kotnik@mapef.pefri.hr
}

Our high-resolution optical spectroscopic survey of the symbiotic star CH Cyg, extending over the quiescent period $1987-1989$ between two outbursts, shows that the spectrum did not completely lose its symbiotic character. Moreover, signs of radiation of the hot source were present, not only as remnants from the previous outburst, but also as an indication of a new one. Some of the evidences which support this idea are following:

- Variation of excitation and vibration temperatures of neutral metals and TiO moleculs respectively

- Untypical microturbulent velocity dependance of the excitation potential of the lower state for $\mathrm{Fe} I$

- Double-peaked Balmer profiles of $\mathrm{Ha}$ and $\mathrm{Hb}$ with temporary disappearance of the central absorption

- Presence of forbbiden emission lines [FeII] [ SII] [NeIII] together with OIII with complex profile and variable intensity. 